\title{
THE PLANETARY NEBULA PHASE
}

\author{
MANUEL PEIMBERT \\ Instituto de Astronomía, UNAM. \\ Apdo.P. 70-264, C.P. 0ł510, \\ México, D.F.
}

ABSTRACT. Some recent results on planetary nebulae research are discussed.

\section{The Planetary Nebula Phase as a Main Stage of Stellar Evolution}

Intermediate mass stars (IMS), those with masses in the $0.8 \leq M_{i} / M_{\odot} \leq 8$ range where $M_{i}$ is their initial mass, are considered to be progenitors of planetary nebulae (e.g. Peimbert 1990a and references therein).

The birth rate of PN per unit luminosity, $\dot{\xi}$, has been estimated for the Galaxy from the $M_{b o l}$ and the $(B-V)_{0}$ versus $\dot{\xi}$ relationships for nearby galaxies and amounts to: $\dot{\xi}=(12 \pm 3) \times 10^{-12} \mathrm{yr}^{-1} L_{\odot}^{-1}$ (Peimbert 1990b). Renzini and Buzzoni (1986) computed the stellar death rate, $\dot{S}$, for galaxies as a function of age and for three widely different initial mass functions; from these computations it is found that, for the Galaxy, the stellar death rate for stars in the $0.8 \leq M_{i} / M_{\odot} \leq 8$ range amounts to: $\dot{S}=(18 \pm 3) \times 10^{-12}$ $\mathrm{yr}^{-1} L_{\odot}^{-1}$ (Peimbert $1990 \mathrm{~b}$ ). The $\dot{\xi}$ and $\dot{S}$ values imply that most of the IMS go through the PN phase.

From the Cudworth (1974) distance scale and the local birth rate of PN it is found that $\dot{\xi}=9.8 \times 10^{-12} \mathrm{yr}^{-1} L_{\odot}^{-1}$. Alternatively from the Cahn and Kaler (1971) distance scale and the local birth rate of PN it is found that $\dot{\xi}=48.2 \times 10^{-12} \mathrm{yr}^{-1}$ $L_{\odot}^{-1}$ (Peimbert 1990b). These $\dot{\xi}$ values compared with the $\dot{\xi}$ and $\dot{S}$ values mentioned above imply that long distance scales to PN, like those of Cudworth (1974) or Mallik and Peimbert (1988), are favored over shorter ones, like those of Cahn and Kaler (1971) or Daub (1982).

The white dwarf local birth rate per unit luminosity derived by Fleming et al. (1986) and Downes (1986) amounts to: $\dot{\xi}(W D)=(6 \pm 3) \times 10^{-12} \mathrm{yr}^{-1} L_{\odot}^{-1}$. This result also implies that most of the IMS go through the PN phase and that long distance scales to $\mathrm{PN}$ are favored over shorter ones.

\section{Chemical Abundance Determinations}

There have been a large number of papers and reviews on chemical abundance determinations (e.g. Peimbert 1990a and references therein, Aller 1990, Kaler et al. 1990, Kaler and Jacoby 1990, Henry 1990, Maciel 1991, Clegg 1991, Perinotto 1991, Dopita 
and Meatheringham 1991). These results have been used to: a) compare the observed abundances with those predicted by stellar evolution models, b) determine the abundance gradients across the disk of the Galaxy, the pregalactic helium abundance and the $\Delta Y / \Delta Z$ ratio, c) estimate the $\mathrm{He}, \mathrm{C}$ and $\mathrm{N}$ enrichment of the interstellar medium and $\mathrm{d}$ ) study the evolution of the halo of the Galaxy. From these studies it has been found that $\mathrm{ON}$ cycling is needed to explain the very large $\mathrm{N} / \mathrm{O}$ ratios and the $\mathrm{N} / \mathrm{O}$ versus $\mathrm{O} / \mathrm{H}$ anticorrelation derived from type I PN (see also Feibelman et al. 1985) and that type I PN originate from more massive progenitors than other types of PN (see also Stasinska and Tylenda 1990).

\section{Halo Planetary Nebulae}

A recent review on the abundances of ten halo PN, type IV PN, has been presented by Peimbert (1991); about half of them show excesses in O/Ar and Ne/Ar relative to the solar neighborhood values, these excesses could be due to their progenitor stars or to galactic chemical evolution.

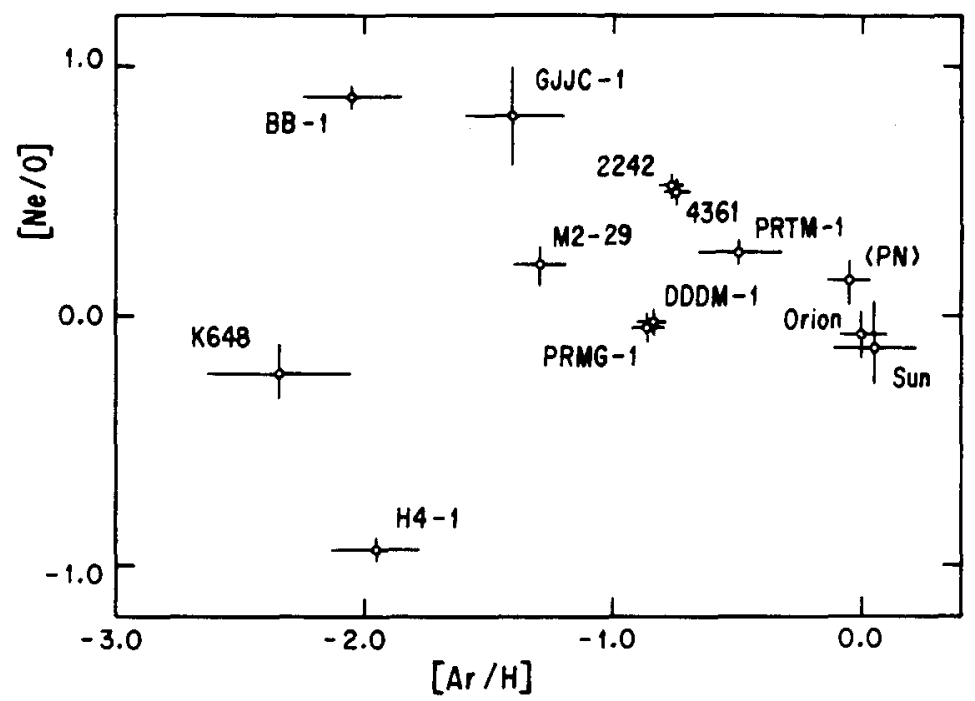

Fig. 1. Plot of $[\mathrm{Ne} / \mathrm{O}]$ versus $[\mathrm{Ar} / \mathrm{H}]$ abundances for halo $\mathrm{PN}$, where $[\mathrm{A} / \mathrm{B}]=\log (\mathrm{A} / \mathrm{B})-\log (\mathrm{A} / \mathrm{B})_{\text {pop } I}$. We have also plotted the abundances for the Sun, the Orion nebula and an average for type II PN.

From the computations of Thielemann et al. (1991) it is found that the yields of a $25 M_{\odot}$ star are very different from those of a $13 M_{\odot}$ star; the $\mathrm{O} / \mathrm{Ar}$ and $\mathrm{Ne} / \mathrm{Ar}$ yields are higher by more than an order of magnitude in the $25 M_{\odot}$ than in the $13 M_{\odot}$ model. Therefore it is conceivable that early in the evolution of the halo, stars with $M \sim 25 M_{\odot}$ contributed more to the enrichment of the interstellar medium than stars with $M \sim 13 M_{\odot}$. Moreover it is also possible that, due to poor mixing in the ISM, some stars could have been made of $\mathrm{Ne} / \mathrm{Ar}$ and $\mathrm{O} / \mathrm{Ar}$ rich material.

Alternatively the $\mathrm{Ne} / \mathrm{O}$ range predicted by the models of Thielemann et al. (1991) amounts to about a factor of two, while the observed range comprises about two orders of magnitude (see Figure 1); consequently part of the observed Ne/O range seems to be due to nuclear reactions in the PN progenitors and not to galactic chemical evolution. 
The Ne/O defficiency in $\mathrm{H} 4-1$ indicates production of $\mathrm{O}$ by the progenitor star. The Ne/O excesses in BB-1 and GJJC-1, the PN in the globular cluster M22, imply production of $\mathrm{Ne}$ by the progenitor stars. The $\mathrm{Ne} / \mathrm{O}$ ratio in $\mathrm{K648}$, the $\mathrm{PN}$ in the globular cluster M15, is similar to: a) the $\mathrm{H} \Pi$ regions ratio (Meyer 1989) b) the average PN ratio (Kaler 1978, Henry 1989) and c) the ratio of the Ne and O yields predicted by Thielemann et al. (1991); for $\mathrm{K} 648$ the $\mathrm{O} / \mathrm{Ar}$ and $\mathrm{Ne} / \mathrm{Ar}$ excesses could be due to galactic chemical evolution but they could also be due to the progenitor star.

\section{References}

Aller, L.H. 1990, Pub. A.S.P., 102, 1097.

Cahn, J.H. and Kaler, J.B. 1971, Ap. J.S.S., 22, 319.

Clegg, R.E.S. 1991, in IAU Symp. 145, Evolution of Stars: The Photospheric Abundance

Connection, eds. G. Michaud and A. Tutukov (Dordrecht: Kluwer), p. 387.

Cudworth, K.M. 1974, A.J., 79, 1384.

Daub, C.T. 1982, Ap. J., 260, 612.

Dopita, M.A., and Meatheringham, S.J. 1991, Ap. J., 377, 480.

Downes, R.A. 1986, Ap. J.S.S., 61, 569.

Feibelman, W.A., Aller, L.H., Keyes, C.D., and Czyzak, S.J. 1985, Proc. N.A.S., 82, 2202.

Fleming, T.A., Liebert, J., and Green, R.F. 1986, Ap. J., 308, 176.

Henry, R.B.C. 1989, M.N.R.A.S., 241, 453.

Henry, R.B.C. 1990, Ap. J., 356, 229.

Kaler, J.B. 1978, Ap. J., 225, 527.

Kaler, J.B., and Jacoby, G.H. 1990, Ap. J., 362, 491.

Kaler, J.B., Shaw, R.A., and Kwitter, K.B. 1990, Ap. J., 359, 392.

Maciel, W.J. 1991, in Elements and the Cosmos, eds. R. Terlevich et al. (Cambridge Univ. Press), in press.

Mallik, D.C.V., and Peimbert, M. 1988, Rev.Mex.A.A., 16, 111.

Meyer, J.-P. 1989, in Cosmic Abundances of Matter, ed. C.J. Waddington (New York:

Amer. Inst. Phys.), p. 245.

Peimbert, M. 1990a, Rep. Prog. Phys., 53, 1559.

Peimbert, M. 1990b, Rev.Mex.A.A., 20, 119.

Peimbert, M. 1991, in Elements and the Cosmos, eds. R. Terlevich et al. (Cambridge

Univ. Press), in press.

Perinotto, M. 1991, Ap.J.S.S., 76, 687.

Renzini, A., and Buzzoni, A. 1986, in Spectral Evolution of Galaxies, ed. C. Chiosi and A. Renzini (Dordrecht: Reidel), p. 195.

Stasinska, G., and Tylenda, R. 1990, A.A., 240, 467.

Thielemann, F.-K., Nomoto, K., and Hashimoto, M. 1991, in Supernovae, eds. J. Audouze et al. (Elsevier Science Publishers), in press. 